\title{
Changes of clinical activities in an orthopaedic institute in North Italy during the spread of COVID-19 pandemic: a seven-week observational analysis
}

\author{
Luigi Zagra ${ }^{1}$ (D) Martina Faraldi ${ }^{2}$ (D) $\cdot$ Fabrizio Pregliasco $^{3}$ (D) $\cdot$ Anna Vinci $^{3} \cdot$ Giovanni Lombardi $^{2,4}$ (D) \\ Immacolata Ottaiano ${ }^{5} \cdot$ Riccardo Accetta $^{6} \cdot$ Paolo Perazzo $^{7} \cdot$ Rocco D'Apolito $^{1}$ (I)
}

Received: 21 April 2020 / Accepted: 27 April 2020 / Published online: 24 May 2020

(C) SICOT aisbl 2020

\begin{abstract}
Purpose The COVID-19 pandemic is importantly affecting the orthopaedic practice all over the world with Northern Italy being the first European area that faced the worst scenario. In this study, the changes in clinical practice occurred in an orthopaedic center in Milan are described.

Methods Number and type of admissions, outpatients cancelled and preserved, emergency room, and intensive care unit activities have been analyzed in the timeframe of seven weeks since the beginning of the pandemic (from February 24th to April 10th) and compared with the same period in 2019.

Results The planned surgical admissions declined from 2172 in 2019 to 664 in $2020(69.42 \%, p<0.0001)$, while emergencies increased from 158 to 268 (69.62\%). The rehabilitation admissions declined from 414 to 69 (83.33\%). The overall admission decreased by $63.52 \%$, the trend showed a drop in the last weeks. Surgery performed in the COVID-19 operating room increased by $16.7 \%$ in the last week. Seven deaths occurred $(0.7 \%$ of all orthopaedics and trauma admissions) compared with four $(0.1 \%)$ which happened in the same period in 2019 ( $p=0.004)$. Six of these patients were suffering from COVID-19. A total of 23,580 outpatients (93.8\%) were cancelled. Emergency room consultations declined by $68.14 \%$ and $63.47 \%$ among white and green priority, respectively, while increased by $25 \%$ and $100 \%$ among yellow and red, respectively.

Conclusion These numbers show the radical changed scenario in an orthopaedic center in Milan during COVID-19 pandemic. Elective surgery declined rapidly going close to zero, outpatient admissions were restricted to cases that cannot be postponed, while emergencies increased due to the role played by the hospital as referral orthopaedic centre during the pandemic. The still ongoing emergency will have important impacts on the overall orthopaedic healthcare management for the next months.
\end{abstract}

Keywords COVID-19 $\cdot$ Coronavirus $\cdot$ Orthopaedic surgery $\cdot$ Orthopaedic trauma $\cdot$ Healthcare systems

Luigi Zagra

luigi.zagra@ fastwebnet.it

1 Hip Department, IRCCS Istituto Ortopedico Galeazzi, Via Riccardo Galeazzi 4, 20161 Milan, Italy

2 Laboratory of Experimental Biochemistry, IRCCS Istituto Ortopedico Galeazzi, Milan, Italy

3 Health Management, IRCCS Istituto Ortopedico Galeazzi, Milan, Italy

4 Department of Athletics, Strength and Conditioning, Poznań University of Physical Education, Poznan, Poland

5 IRCCS Istituto Ortopedico Galeazzi, Milan, Italy

6 ER Department, IRCCS Istituto Ortopedico Galeazzi, Milan, Italy

7 Department of Anesthesiology, IRCCS Istituto Ortopedico Galeazzi, Milan, Italy

\section{Introduction}

The profound healthcare reorganization caused by the coronavirus disease 2019 (COVID-19) pandemic has resulted in a significant reallocation of health resources with the focus on COVID-19 patients all over the world [1].

Northern Italy was the first European area that faced the worst infection scenario. Lombardy is the largest Italian region with a recorded population of $10,060,000$ people at the beginning of 2020 [2]. It is the major economic and industrial area of the country. The Regional Health System is recognized as one of the best performing in Italy including orthopaedic procedures. A total of $23.4 \%$ of all the total joint replacements and $24.0 \%$ of all revisions in Italy are performed in the Lombardy Region according to the Italian Arthroplasty Registry (RIAP) [3]. 
Lombardy has been suffering the most critical novel SARS-CoV2 situation in Italy with a rapid and acute spread of the pandemic during February and March 2020. At the time of data retrieved (April 15th), the total number of COVID-19positive cases in the region was 62,153 , of which 12,043 patients were hospitalized and 1074 more in intensive care units (ICUs) and 11,377 people were dead (57\% of overall Italian deceased) [4].

The rapid pandemic spread has dramatically affected the orthopaedic practice. Hospitals and departments have been quickly converted to COVID-19 care centres since the beginning of the emergency. Staff and operating rooms (ORs) have been converted, elective surgical procedures have been either cancelled or postponed, and nonurgent consultations have been stopped. Most general hospitals have been overwhelmed with SARS-CoV2 patients while, according to the regional reorganization, non-deferrable orthopaedics and trauma cases are being referred to selected centers, designated as either minor or major trauma hubs.

IRCCS Istituto Ortopedico Galeazzi is located in Milan, the capital of Lombardy. It is one of the major orthopaedic centres in Italy with high volume of surgery (4006 total joints procedures and 1759 spine procedure in 2019). It provides emergency service for minor orthopaedic trauma, it is accredited to the Italian Ministry of Health as clinical and basic research center and provides academic and training activity. To cope with the emergency period, the hospital has been designated by the regional government as a referral centre for minor trauma and orthopaedics and has even contributed to COVID-19 patient care in ICU and in a converted department.

The purpose of this study is to describe the profound changes in the clinical practice and the consequent effects on the clinical management of orthopaedic patients that have occurred in a specialized orthopaedic centre during the first seven weeks (from February 24th to April 10th 2020) of COVID19 emergency in Milan.

\section{Materials and methods}

Internal administrative flows integrated and double-checked by the health management data of the hospital represented the data sources of the present study. Approval was obtained by the institutional review board, in using anonymous data flows.

\section{Reorganization of the hospital in a network area}

The trigger of a consecutive series of changes in Italy was the identification of the first Italian citizen affected by severe acute respiratory syndrome coronavirus 2 (SARS-CoV2) in Lombardy. He was a young man from Codogno, a small town in the southern Lombardy on February the 21st. The day after National Government's restrictions fixed some areas of
Lombardy as red zones and closed schools for COVID-19. At this point, Galeazzi Institute started to reduce the operating rooms and the number of elective operations since February 24th (Week 1). The following week, some more surgeries were allowed; in the meantime, the patients with less severe pain conditions started to decline spontaneously their attendance both for surgical procedures and for consultations (Week 2). On March 8th, the number of operating rooms (ORs) was reduced to $60 \%$ (Week 3) in parallel to regional and national lockdown, approved on the same day, and following the regional resolution delivered that established roles and duties of regional hospitals in order to face the spreading of the pandemic. Moreover, during this week, a dedicated COVID-19 OR was quickly set up. On March 14th, the orthopaedic network was rebuilt by an addendum to the regional resolution. Galeazzi Institute was identified as a hub for nondeferrable orthopaedic procedures (Table 1) and for minor orthopaedic trauma, while most of general hospitals and ICUs in the region were increasingly overloaded with COVID-19 patients. Since March 16th (Week 4), nearly all the surgery but acute trauma, acute infections, and malignant tumours resections were stopped in our institution. Also, cases to be, theoretically, performed according to regional rules (i.e. severe loosening or recurrent dislocations or rapid developing arthritis with bone necrosis) have been stopped as the widespread dissemination of COVID19 infections increased the risk of nosocomial infection. Since Week 4, only two or three ORs were running depending on the number of emergencies. Hence, during Weeks 5, 6, and 7, only trauma, acute orthopedic infections, and malignant tumours resections were performed both on COVID-19 positive and negative patients.

Reorganization of the institute went on consequently. Starting from Week 3, departments were isolated for COVID-19 patients, with healthcare personnel provided with high-level personal protection equipment (PPE), impossibility for relatives to meet patients, according to infection disease protection protocols. During Week 4, in order to face the huge increase in the number of infected patients all around the region, even non-orthopedic COVID-19 patients were admitted to the hospital and were allocated in a separate department. Starting from Week 5, even the relatives of SARS-CoV2negative patients were no longer allowed to access the hospital

Table 1 Orthopaedic surgery authorized by Regional Authorities (out of trauma) since March 14th

\footnotetext{
- Septic arthritis

- Malignant tumours

- Benignant tumours at risk of fracture

- Neurologic deficit

- Rapidly progressive arthritis (bone necrosis)

- Acute tendon lesions

- TJA dislocations and severe loosening
} 
Table 2 Overall admissions 2020 vs same 7-week period 2019

\begin{tabular}{lllllll}
\hline Year & $\begin{array}{l}\text { Type of } \\
\text { admissions }\end{array}$ & $\begin{array}{l}\text { No. per type of } \\
\text { admissions }\end{array}$ & $\begin{array}{l}\text { No. of } \\
\text { admissions }\end{array}$ & $\begin{array}{l}\% \text { on no. of } \\
\text { admissions }\end{array}$ & $\begin{array}{l}\chi^{2} \text { test } \\
\mathrm{df}(1)\end{array}$ & $p$ value \\
\hline 2019 & Surgery & 2330 & 2744 & 84.90 & 43.84 & $<0.0001$ \\
& Rehabilitation & 414 & 15.10 & & \\
2020 & Surgery & 932 & 1001 & 93.10 & & \\
& Rehabilitation & 69 & & 6.90 & & \\
\hline
\end{tabular}

This table contains data of surgery and rehabilitation admissions in 2019 and 2020 (from February 24th to April 10th), considering the same period. Percentage of each type of admission was also presented. Pearson's Chisquare test and relative $p$ value were performed to compare the different types of admission between 2019 and 2020. $p<0.05$ was considered statistically significant in order to further reduce the risk of infection. Since Week 5 , all the hospitalized patients have been screened for COVID19 infection with nasopharyngeal swab and allocated at time of admission in an observational area until the result of the test (usually taking 1 day) and then transferred to the COVID-19 department or to the non-COVID ones who were located on separate floors. Due to these restrictions and to the reduced nurse staff (in the meantime, the number of personnel in quarantine raised), the number of beds was reduced to less than a third (median 100; 364 beds including rehabilitation are usually occupied).

A special effort involved the anaesthetists of the institution. Usually the ICU is dedicated to post-operative monitoring and intensive care of the most complicated patients and those undergoing most complex surgical procedures. Six out of eight beds are usually occupied, every day, for this activity. Starting from Week 3, four beds were turned in an isolated area for severe intubated COVID-19 patients coming from other hospitals. During the most critical time in Week 4, as part of the Regional Network of ICUs, the ICU was extended and moved into a floor usually fully occupied by an operating block that was adapted for respiratory assistance of ten intubated patients.

Number and type of admissions in the hospital are reported in a seven week period since the beginning of the pandemic phase (from February 24th to April 10th) and compared with the same period in 2019. The analysis includes type of surgeries and number of deaths in COVID-19 and non-COVID-19 orthopedic patients, number of outpatients canceled and preserved, and frequency of patients in emergency room.

\section{Statistical analysis}

The differences among the data observed over the seven weeks in 2020 and the corresponding period in 2019 were analyzed by means of the Mann-Whitney $U$ test. Data recorded in the first three weeks and in the last four weeks of the seven week period in 2019 and 2020 have been compared using ordinary two-way ANOVA with Sidak's multiple comparison test. Data have been analyzed by the means of $\chi^{2}$ test in order to compare the corresponding weeks in 2019 and 2020: admission rates in terms of surgery cases and rehabilitation cases, planned vs urgent surgery, and number of deaths. Statistical analysis has been performed with GraphPad Prism v6.0 (GraphPad Software, Inc., La Jolla, CA, USA).

\section{Results}

\section{Number and type of surgery and admissions}

The overall number of admissions declined from 2744 in 2019 to 1001 in $2020(63.52 \%$, Tables 2,3 , and 4$)$ in the whole period, but the trend shows a major drop of the curve on Week 4 (Fig. 1a). The mean value of total admissions in the seven observed weeks was decreased in 2020 compared with 2019 ( $p=0.0006)$, as shown in Fig. 1b. The decrease continued up to $88 \%$ during Week 7 with only 47 admissions (4 oncologic cases, 41 trauma patients, and 3 others included 1 tendon rupture and 2 infections) (Table 3 ). The mean value of admissions in the first three weeks and the last four weeks in 2020
Table 3 Admissions by weeks comparing 2019 and 2020

\begin{tabular}{llllllll}
\hline Year & Week 1 & Week 2 & Week 3 & Week 4 & Week 5 & Week 6 & Week 7 \\
\hline 2019 & 398 & 376 & 415 & 370 & 388 & 418 & 379 \\
2020 & 206 & 335 & 204 & 79 & 66 & 64 & 47 \\
Ratio 2020/2019(\%) & 51.76 & 89.10 & 49.16 & 21.35 & 17.01 & 15.31 & 12.40 \\
& $62.66 \%$ & & & $16.46 \%$ & & & \\
\hline
\end{tabular}

This table contains data of number of admissions in 2019 and 2020, considering the same period (from February 24th to April 10th). Ratio between admission of 2020 and 2019 and difference between admissions of 2020 and 2019 by weeks and by the first 3 weeks and the last 4 weeks are presented 
Table 4 Planned vs emergency admissions in 2019 and 2020

\begin{tabular}{lllllll}
\hline Year & $\begin{array}{l}\text { Type of } \\
\text { admissions }\end{array}$ & $\begin{array}{l}\text { No. per type of } \\
\text { admissions }\end{array}$ & $\begin{array}{l}\text { No. of } \\
\text { admissions }\end{array}$ & $\begin{array}{l}\% \text { on no. of } \\
\text { admissions }\end{array}$ & $\begin{array}{l}\chi^{2} \text { test } \\
\mathrm{df}(1)\end{array}$ & $p$ value \\
\hline 2019 & Planned & 2172 & 2.330 & 93.20 & 283.10 & $<0.0001$ \\
& Urgent* & 158 & & 6.80 & & \\
2020 & Planned & 664 & 932 & 71.20 & & \\
& Urgent* & 268 & & 28.80 & & \\
\hline
\end{tabular}

This table contains data of planned and urgent surgery in 2019 and 2020, considering the same period (from February 24th to April 10th). Percentage of each type of admission is also presented. Pearson's Chi-Square test and relative $P$ value were performed to compare the different types of admission between 2019 and $2020 . P<0.05$ was considered statistically significant
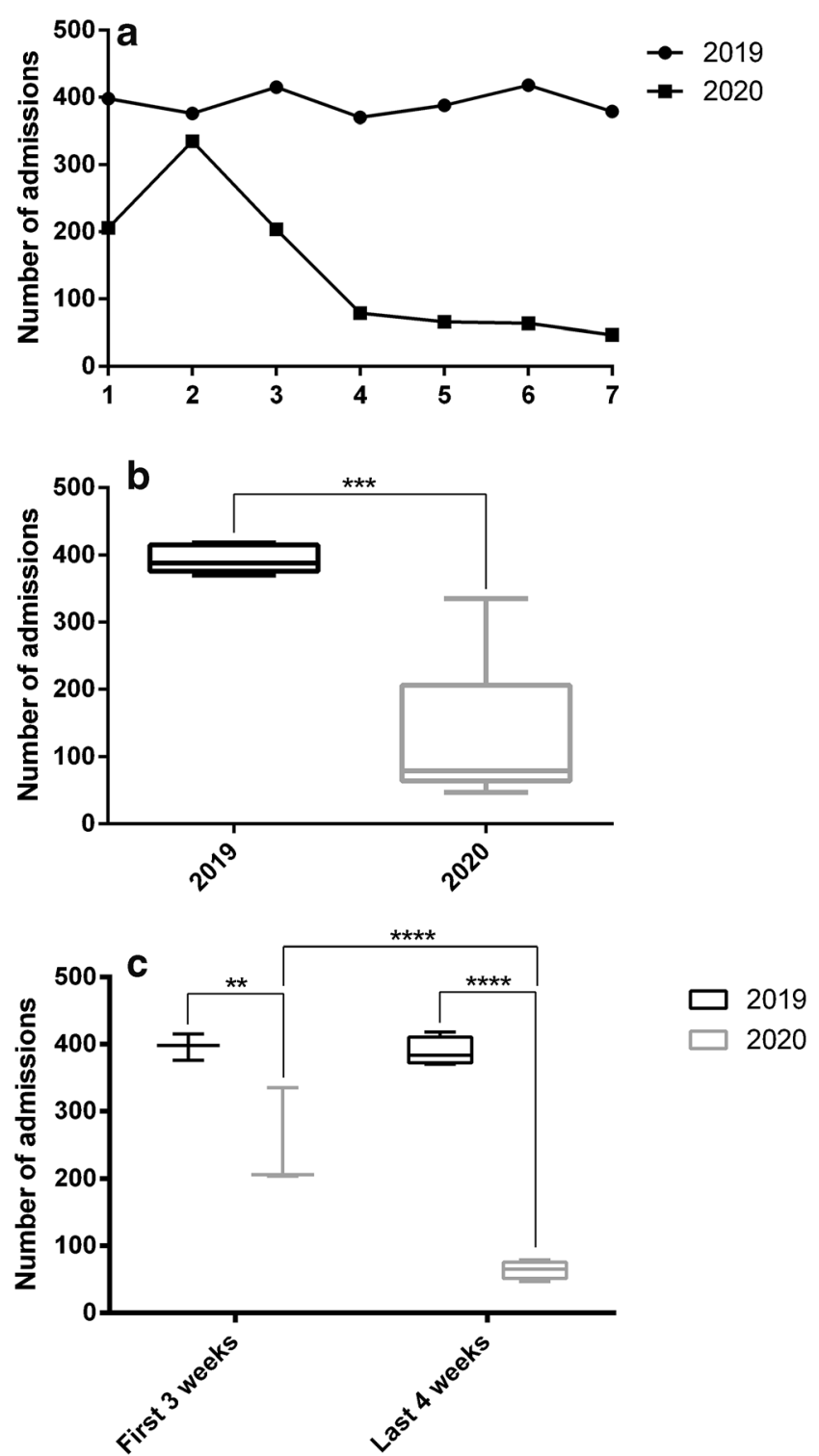

Fig. 1 Overall admissions from February 24th to April 10th 2020 compared with the same period in 2019. a Trend of admissions during the 7 weeks. b Mean value of admissions 2020 vs 2019. c Mean value of admissions in the first 3 weeks and in the last 4 weeks in 2020 compared with 2019 were both significantly decreased compared with 2019 ( $p=$ $0.0013, p<0.0001)$. Moreover, considering the mean number of admissions in the first three weeks and the last four weeks (Fig. 1c), the two values were comparable $(p=0.96)$ in 2019 , while they significantly differed in 2020 ( $p=0.0001)$. In addition, the rehabilitation admissions declined from 414 to 69 $(83.33 \%)$ (Table 2). The rehabilitation department was closed at the end of Week 4 due to the decreased number of patients and also to limit at the maximum the risk of nosocomial infection spreading while the hospital was reorganized to host non-orthopedic COVID-19 patients, on a separate floor. The planned surgical admissions declined from 2172 in 2019 to 664 in 2020 (69.42\%), whereas emergencies increased from 158 (6.8\% of admissions) to 268 (28.8\% of admissions) in $2020(69.62 \%)$, including network admissions and transfers from other hospitals (Table 4). Operations performed in the COVID-19 OR were 31 (3.5\% in the whole period), raising up to $16.67 \%$ (6 cases vs 36 in standard ORs) in the Week 7 (Table 5). Basically, the activity has been converted from mainly elective surgery to emergencies.

\section{Outpatient activity}

A total number of 23,580 (93.8\%) outpatients were canceled declining to 365 in the Week 7 (Table 6), and the mean value of outpatients in all period of 7 weeks in 2020 significantly decreased compared with 2019 ( $p=0.03$ ) (Fig. 2a). Starting from Week 2, a spontaneous reduction of the number of outpatients due to the regional lockdown and to the increasing concerns of the population on the pandemic was observed. During Week 4, consultations were cancelled directly by the booking office of the hospital and then limited to nondeferrable cases (sutures, plaster cast removals, immediate postoperative controls, emerging complications) based on an agreement between the call centre and the surgeons. This situation has continued along the time (Weeks 5, 6, and 7). Indeed, in 2020, the mean value of outpatient in the last four weeks decreased in comparison with $2019(p<0.0001)$. Moreover, in 2020, the mean value of outpatients in the last 
Table 5 Number of operations performed in COVID-19 OR compared with standard ORs along the 7 weeks

\begin{tabular}{llllllll}
\hline Type of surgery & Week 1 & Week 2 & Week 3 & Week 4 & Week 5 & Week 6 & Week 7 \\
\hline COVID-19+ & 2 & 5 & 2 & 6 & 8 & 2 & 6 \\
COVID-19- & 180 & 296 & 166 & 66 & 54 & 54 & 36 \\
Ratio COVID+/- (\%) & 1.11 & 1.69 & 1.20 & 9.09 & 14.81 & 3.70 & 16.67 \\
& $1.40 \%$ & & & $10.48 \%$ & & & \\
\hline
\end{tabular}

This table contains data of operations (COVID+ and COVID-) of 2020, considering the period from February 24th to April 10th. Ratio between COVID+ and COVID- cases

*Includes ambulance emergency network admissions, ER admissions, and transfers from other hospitals four weeks significantly decreased compared with the outpatients in the first three weeks $(\mathrm{p}<0.0001)$, as shown in Fig. $2 \mathrm{~b}$.

\section{The flow of patients in emergency room}

The flow of patients in the emergency room (ER) spontaneously reduced, mainly in the less severe cases. All the patients were admitted to triage procedures as usual, the only differences were a strict protocol regarding PPE of the staff and the number of patients admitted in the waiting room. Moreover, no relatives were allowed and protection devices were provided to the patients. According to triage classification when compared with 2019 (Table 7), there was a reduction of $68.14 \%$ and $63.47 \%$ in white and green codes, while yellow codes increased by $25 \%$ and red codes went up from 0 in 2019 to 7 patients in 2020 (Fig. 3). ERs in orthopedic centers are not usually intended as a life-threatening first aid.

\section{The impact of pandemic on hospital contamination and mortality}

A total number of 79 COVID-19 patients were treated; 25 (31.64\%) of them were orthopaedics or trauma cases that developed SARS-CoV2-like symptoms during hospitalization (3\% of all admissions) in the surgical or in the rehabilitation department. They were preventively isolated in an observational area and, after the test, recognized as COVID-19positive and sent in the COVID-19 department. Sixteen more were orthopaedics and trauma patients recognized as positive since the admission. The remaining 38 were not orthopaedics or trauma patients: two were admitted directly by the ER for respiratory symptoms and 36 were transferred from other hospitals in the COVID-19 department or in the ICU that was converted to host positive patients, as previously described. The ICU for COVID-19 had 15 admissions in total, with four deaths and four discharges.

Eleven patients died in the study period at our hospital. Seven deaths occurred $(0.7 \%$ of all admissions) among orthopaedics and trauma patients, compared with four $(0.1 \%)$ in the same period of $2019(p=0.004)$ (Table 8$)$, with six of these patients suffering from COVID-19 (Table 9). The seven cases included four patients with femoral neck fractures (three COVID-19 patients) aged 77, 87, 88, and 95 years old, respectively, all females, one oncologic patient with bone metastasis (74 years old, male), and two women who underwent total knee arthroplasty ( 79 and 84 years old, respectively) who gave a positive swab test during the rehabilitation time and developed pulmonary complications related to COVID-19 infection. Four deaths occurred in COVID-19 positive patients with no orthopaedic-related problems, coming from other hospitals.

\section{Discussion}

The aim of this study was to describe the profound modifications of the SARS-CoV2 pandemic. To the best of our knowledge, this is the first report about the change in orthopaedic practice in a specialized orthopaedic centre during the spread of COVID-19 pandemic over seven weeks of activity and the
Table 6 Number of outpatients 2020 vs 2019 per week

\begin{tabular}{llllllll}
\hline Year & Week 1 & Week 2 & Week 3 & Week 4 & Week 5 & Week 6 & Week 7 \\
\hline 2019 & 8167 & 7974 & 8150 & 8301 & 8488 & 8490 & 8127 \\
2020 & 8670 & 5927 & 6352 & 2692 & 761 & 383 & 365 \\
Ratio 2020/2019(\%) & 106.16 & 74.33 & 77.94 & 32.43 & 8.97 & 4.51 & 4.49 \\
& $86.24 \%$ & & & $12.58 \%$ & & & \\
\hline
\end{tabular}

This table contains data of outpatients in 2019 and 2020, considering the same period (from February 24th to April 10th). Ratio between number of outpatients of 2020 and 2019 and differences between outpatients of 2020 and 2019 by weeks and by the first 3 weeks and the last 4 weeks are presented 

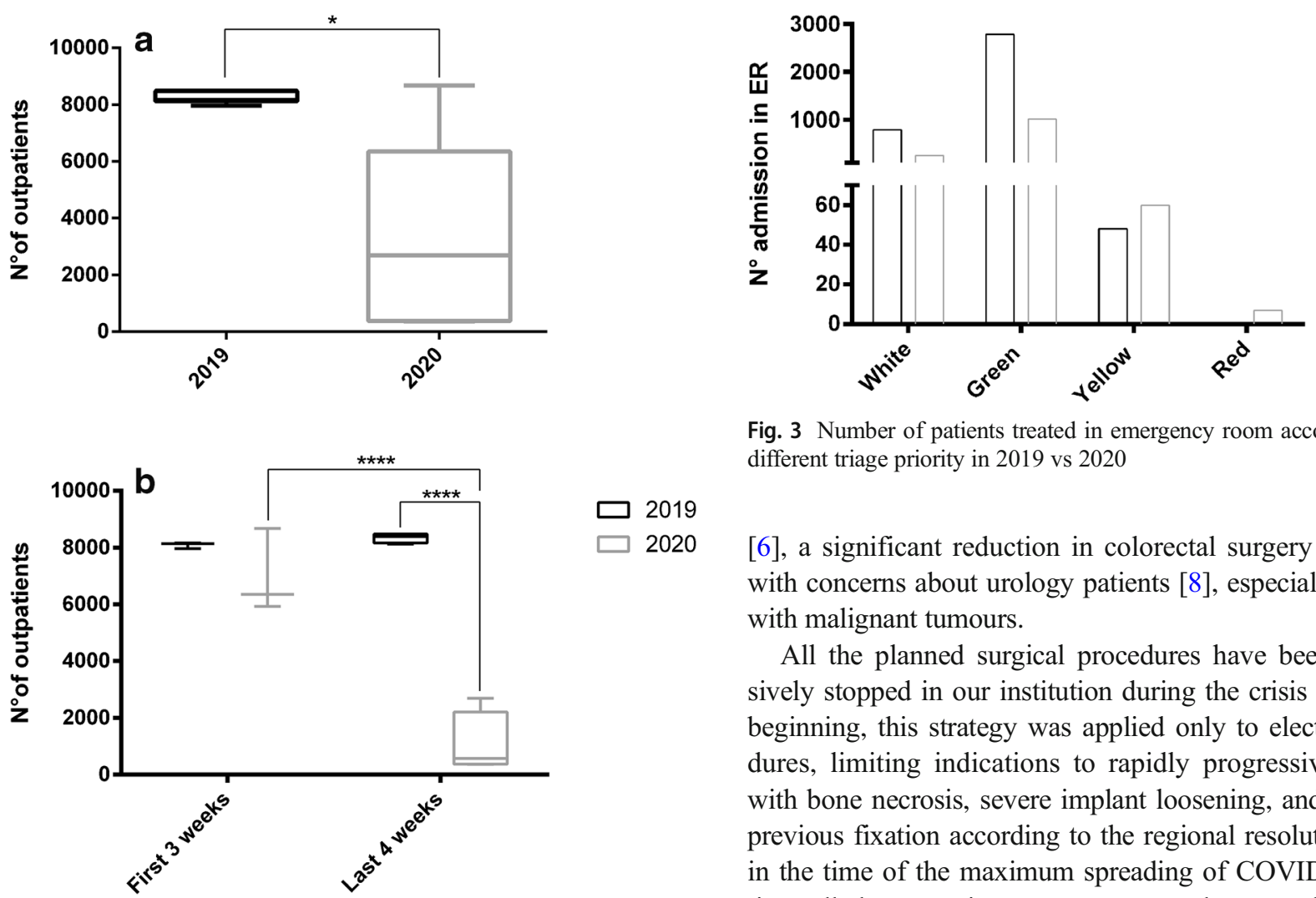

Fig. 3 Number of patients treated in emergency room according to the different triage priority in 2019 vs 2020

[6], a significant reduction in colorectal surgery [7], along with concerns about urology patients [8], especially in those with malignant tumours.

All the planned surgical procedures have been progressively stopped in our institution during the crisis [9]. At the beginning, this strategy was applied only to elective procedures, limiting indications to rapidly progressive arthritis with bone necrosis, severe implant loosening, and failure of previous fixation according to the regional resolution. Then, in the time of the maximum spreading of COVID-19 infection, all the operations were postponed except for trauma, acute infection, and malignant tumours resections. This type of approach taken out by both health authorities and orthopaedic surgeons has several undoubtable reasons: to preserve resources for COVID-19 patients (staff, hospital beds, ICU, economy), to reduce the risks of contamination (of the staff, of the patients, of the whole community) in an "open environment" such as the hospital that is difficult to be controlled, and to guarantee a safe and effective rehabilitation time for the patients operated upon. The possible contamination rate in all the admissions was 3\%, extremely low; nevertheless, old fragile patients are at major risk. For this reason, it looks reasonable to stop all the surgery "that can be

Table 7 Number of admissions in emergency room 2020 vs 2019 according to triage classification (code white: lighter case suitable for an office consultation and should not require an emergency treatment; red code: the most severe life threatening)

\begin{tabular}{|c|c|c|c|c|c|c|}
\hline \multirow[t]{2}{*}{ Triage code } & \multirow[t]{2}{*}{ Description } & \multirow[t]{2}{*}{2019} & \multirow[t]{2}{*}{2020} & \multicolumn{2}{|c|}{$\begin{array}{l}\text { Incidence on } \\
\text { total cases }(\%)\end{array}$} & \multirow[t]{2}{*}{ Ratio $2020 / 2019(\%$} \\
\hline & & & & 2019 & 2020 & \\
\hline White & Not urgent problem or of minimal clinical relevance & 791 & 252 & 21.80 & 18.90 & 31.86 \\
\hline Green & $\begin{array}{l}\text { Stable condition without evolutionary risk that usually requires simple } \\
\text { single-specialist therapeutic diagnostic performance }\end{array}$ & 2782 & 1016 & 76.80 & 76.10 & 36.52 \\
\hline Yellow & $\begin{array}{l}\text { Risk of compromising vital functions. Condition with evolutionary } \\
\text { risk or severe pain }\end{array}$ & 48 & 60 & 1.30 & 4.50 & 125.00 \\
\hline Red & Interruption or compromise of one or more vital functions & 0 & 7 & 0.00 & 0.50 & \\
\hline Total & & 3621 & 1335 & & & 36.87 \\
\hline
\end{tabular}

Ratio and differences for patients admitted according to triage classification between 2020 and 2019 are shown 
Table 8 Deaths in orthopaedic and trauma patients 2019 vs 2020

\begin{tabular}{llllll}
\hline Year & No. of deaths & Admissions & Mortality rate $(\%)$ & $\chi^{2}$ test df(1) & $p$ value \\
\hline 2019 & 4 & 2.744 & 0.10 & 8.24 & 0.0040 \\
2020 & 7 & 955 & 0.70 & & \\
\hline
\end{tabular}

This table contains the number of deaths in orthopaedic and trauma patients over the same 2 -week period (from February 24th to April 10th). Pearson's Chi-square test and relative $p$ value were performed to compare 2019 and 2020. $p<0.05$ was considered statistically significant postponed." A case by case discussion with the patients and the relatives about pros and cons of delaying surgery has been done in the most critical cases, sharing the decisions with the patients. An overall increase of mortality in trauma and orthopaedics patients was observed compared with the same period in 2019 that was clearly related to COVID-19.

Not only the admissions have been postponed but also consultations and outpatients have been deferred in the vast majority. At the time of writing, no one could know the real impact of the pandemic on the waiting lists, which is supposed to be huge along with the chances of providing timely and safe treatments to our elective surgical cases. The decision on the consultations that cannot be postponed was taken by clinicians in collaboration with the booking office.

The emergency flow was influenced by the regional resolution that assigned the role of hub for minor trauma to the institute. This decision and the consequent actions taken to reorganize the hospital (creation of complete selected COVID-19 journey including separated OR, reinforcement of emergency staff) show how much is important, in the time of such a pandemic, a global strategy and a well-functioning local network able to provide the best and safest healthcare to the patients. The attendance in the ER spontaneously decreased in the lighter cases.

Orthopaedic surgeons changed their practice according to their best skills. Even some arthroplasty surgeons had taken care of trauma cases and part of the staff supported also the care of COVID-19 patients under the supervision of anaesthetists and clinicians. In emergency, 45 nonorthopaedic patients were treated including ICU assistance. The relation with relatives changed as they were not admitted to the hospital. For this reason, a daily phone call, in order to provide information about patients, was scheduled.
This study has several limitations. First of all, its retrospective nature, but it would not have been possible the contrary considering that the emergency was clearly unexpected until some weeks ago. Moreover, our hospital is a specialized orthopaedic centre. For this reason, its role in the whole COVID-19 pandemic scenario was limited compared with large general hospitals in the region where orthopaedics and even trauma activity was completely stopped. Nevertheless, the focus of the paper was to describe the huge changes in the orthopaedic practice during the pandemic. Another limitation is that, according to continuously changing protocols, the patients were screened for COVID-19 only after Week 4, so it may be possible that the number of SARS-CoV2 has been underestimated. Finally, we did not have results of any test at time of discharge for the negative patients and neither information after the hospitalization about changes in their clinical conditions. However, all the patients were in good clinical conditions at the time of discharge, including respiratory function.

In conclusion, the experience in an orthopaedic centre located in the middle of one of the most critical areas worldwide during the COVID-19 pandemic demonstrated how orthopaedic activities could be seriously affected. The experience showed the need for flexibility by healthcare professionals in their tasks in emergency time, the importance of clear and timely protocols, and of coordination at institutional, regional, and national level. When the present emergency will be hopefully over, new challenges are waiting the orthopaedic community with longer waiting lists, the risk of recurrence of the COVID-19 infection, and probably limited healthcare resources. We hope that the efforts experienced by the orthopaedic staff of our institute could be of help for other colleagues facing a similar challenging pandemic in their countries.
Table 9 Deaths in the 2020 seven-week period according to the type of admission and to COVID-19 test result

\begin{tabular}{|c|c|c|c|c|}
\hline \multirow[t]{2}{*}{ COVID-19 test } & \multicolumn{3}{|c|}{ Orthopedic cases } & \multirow{2}{*}{$\begin{array}{l}\text { Non-orthopedic cases } \\
\text { Total no. of deaths }\end{array}$} \\
\hline & Planned & Urgent & Total no. of deaths & \\
\hline COVID+ at admission & 0 & 2 & 2 & 4 \\
\hline COVID- at admission & 0 & 1 & 1 & 0 \\
\hline COVID+ during admission & 3 & 1 & 4 & 0 \\
\hline Total deaths & 3 & 4 & 7 & 4 \\
\hline
\end{tabular}


Availability of data and material Not applicable

Code availability Not applicable

Authors' contributions All authors contributed to this manuscript, and they all approved the final version.

\section{Compliance with ethical standards}

Conflicts of interest The authors declare that they have no conflict of interest.

Ethics approval A statement is reported in the manuscript with regard to Institutional Review Board Approval.

Consent to participate Not applicable

Consent for publication Not applicable

\section{References}

1. Tsai J, Wilson M (2020) COVID-19: a potential public health problem for homeless populations. Lancet Public Health 5:e186-e187. https://doi.org/10.1016/S2468-2667(20)30053-0

2. Popolazione residente al $1^{\circ}$ gennaio. http://dati.istat.it/Index.aspx? QueryId=18460. Accessed 15 Apr 2020
3. (2017) Quarto Report RIAP 2017. In: RIAP. http://riap.iss.it/riap/it/ attivita/report/2017/12/15/quarto-report-2017/. Accessed 14 Apr 2020

4. Salute M della Covid-19, i casi in Italia 13 aprile ore $18 . \mathrm{http}: / / \mathrm{www}$. s a lut e.gov.it/portale/nuovocoronavirus/ dettaglioNotizieNuovoCoronavirus.jsp?lingua=italiano\&menu= notizie $\& \mathrm{p}=$ dalministero $\& \mathrm{id}=4459$. Accessed $14 \mathrm{Apr} 2020$

5. Chang Liang Z, Wang W, Murphy D, Po Hui JH (2020) Novel coronavirus and orthopaedic surgery: early experiences from Singapore. J Bone Joint Surg Am. https://doi.org/10.2106/JBJS.20. 00236

6. Angelico R, Trapani S, Manzia TM et al (2020) The COVID-19 outbreak in Italy: initial implications for organ transplantation programs. Am J Transplant. https://doi.org/10.1111/ajt.15904

7. Lisi G, Campanelli M, Spoletini D, Carlini M (2020) The possible impact of COVID-19 on colorectal surgery in Italy. Color Dis. https://doi.org/10.1111/codi.15054

8. Connor MJ, Winkler M, Miah S (2020) COVID-19 pandemic - is virtual urology clinic the answer to keeping the cancer pathway moving? BJU Int. https://doi.org/10.1111/bju.15061

9. D'Apolito R, Faraldi M, Ottaiano I, Zagra L (2020) Disruption of arthroplasty practice in an orthopedic center in Northern Italy during the coronavirus disease 2019 pandemic. J Arthroplasty. https://doi. org/10.1016/j.arth.2020.04.057

Publisher's note Springer Nature remains neutral with regard to jurisdictional claims in published maps and institutional affiliations. 